\title{
Unilateral Olivary Degeneration Secondary to Brainstem Cavernous Angioma
}

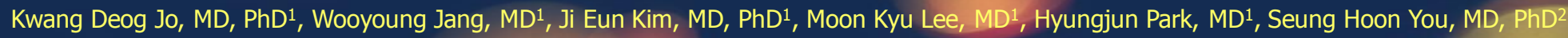
Department of Neurology ${ }^{1}$, Neurosurgery², Gangneung Asan Hospital, University of Ulsan College of Medicine, Gangneung, Korea

\section{Background}

- Hypertrophic olivary degeneration (HOD) is usually caused by a disruption of the dentato-rubro-olivary pathway, also known as the triangle of Guillain-Mollaret.

- The triangle of Guillain-Mollaret is involved in fine voluntary motor control and consists of both the inferior olivary nucleus an the red nucleus on one side and the contralateral dentate nucleus (Fig. 1) ${ }^{1}$.

- HOD is a unique form of degeneration because it results in enlargement of the affected structure rather than atrophy.

- We present a case of unilateral atrophic olivary degeneration secondary to brainstem cavernous angioma.

\section{Case}

- A 55-year-old man was admitted to gastrointestinal department due to a acute gastric ulcer.

- He also complained of constant symptoms of dysarthria and clumsiness of the right hand over the past 12 years.

- He had a history of hypertension and smoking.

- Neurologic examination showed moderate dysarthria and mild clumsiness of fine finger movements in the right hand.

- There was no palatal myoclonus on the 8 days follow up period.

- Brain CT angiogrpahy performed 4 years after the onset of symptoms, and showed slight hyperdense lesion without mass effect and normal vessels of the circle of Willis.

- The first brain MRI was performed 4 years after the onset of symptoms, and MRI showed a $2.5 \times 2 \mathrm{~cm}$, multinodular mass lesion on the right side of the midbrain and pons tegmentum.

- T2 weighted images showed papcorn-like central area of mixed signal intensity with peripheral rim of decreased signal intensity.

- A Follow-up MRI obtained 8 years later showed no significant interval changes of cavernous angioma of the brainstem except a tiny T2 weighted low signal lesion in the right inferior olivary nucleus.

- He was treated with choline alfoscerate and proton pump inhibitors.

\section{References}

1. Ruth Van Eetvelde et al. Imaging features of hypertrophic olivary degeneration. J of the Belgian Soc. of Radiology $2016 ; 100(1): 71,1-6$.
Brain MRI and Angiographic Findings

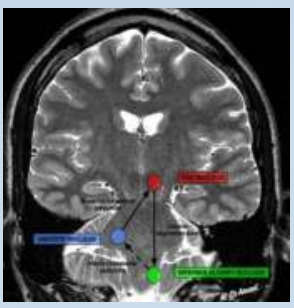

Figure 1.

https://radiopaedia.org/articles/triangle-of-guillain-and-mollaret
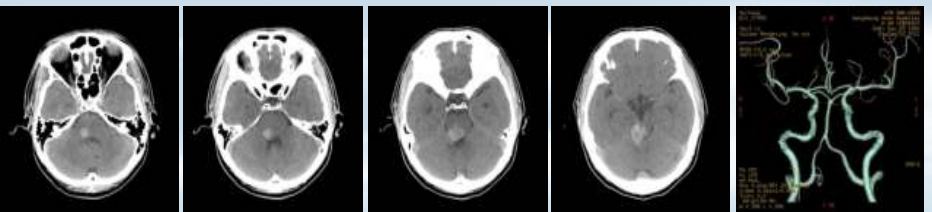

Figure 2. Brain CT angiography of the patient. Brain CT show slight hyperdense lesion in the right tegmentum of the pons and midbrain without mass effect and normal vessels of the circle of Willis.

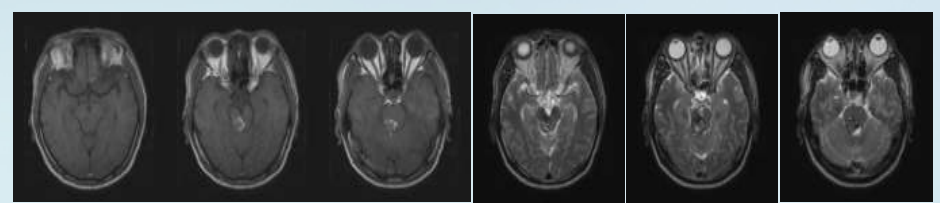

Figure 3. Brain MRI of the patient. MRI show a $2.5 \times 2 \mathrm{~cm}$, multinodular mass lesion on the right side of the midbrain and pons tegmentum. T2 weighted images show A papcorn-like central area of mixed signal intensity with peripheral rim of decreased signal intensity.
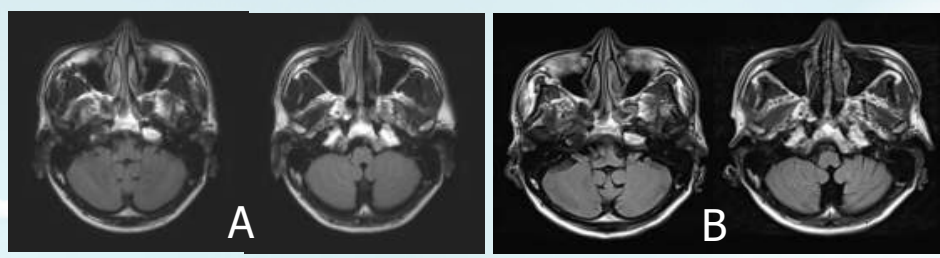

Figure 4. Brain MRI of the patient. Axial T2 weighted images at the level of the medulla show unilateral atrophy in the right medullary olives (B). Axial T2-weighted image at the same level in the same patient but 8 years earlier showed no abnormality (A).

\section{Conclusion}

- We report a case of unilateral olivary degeneration secondary to cavernous angioma of the brainstem.

- We suggest that HOD might be changed to atrophy of the olivay in chronic stage.

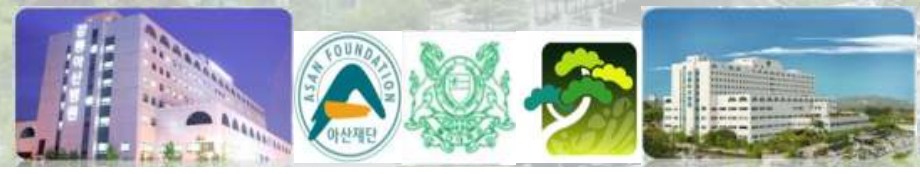

